Revista Brasil. Bot., V.33, n.2, p.325-332, abr.-jun. 2010

\title{
Estrutura genética espacial em populações naturais de Dimorphandra mollis (Fabaceae) na região norte de Minas Gerais, Brasil
}

\author{
ANA CECÍLIA GONÇALVES ${ }^{1}$, CRISTIANE APARECIDA FIORAVANTE REIS², \\ FÁBIO DE ALMEIDA VIEIRA ${ }^{3}$ e DULCINÉIA DE CARVALHO ${ }^{4,5}$
}

(recebido: 23 de julho de 2009; aceito: 01 de abril de 2010)

\begin{abstract}
Spatial genetic structure in natural populations of Dimorphandra mollis (Fabaceae) in the north of Minas Gerais State, Brazil). The knowledge and understanding about the spatial genetic structure are important for management and conservation of forest resources, as well as to evaluate the impacts of exploitation and fragmentation and establish the strategies of sampling in natural populations. We analyzed the spatial genetic structure (SGS) in natural populations of Dimorphandra mollis Benth. to gather information about in situ genetic conservation. A total of ten allozyme polymorphic loci were used to estimate the frequency of 20 alleles in 180 individuals in three natural populations (Vargem da Cruz, Campina Verde and Pau de Fruta) in the north of Minas Gerais State, Brazil. The results revealed high genetic diversity in these populations, showing an average number of alleles per locus $(\hat{A})$ of 2.0. All loci were polymorphic and the average genetic diversity $\left(\hat{H}_{e}\right)$ was 0.463 . The genotypes of $D$. mollis in Vargem Grande and Pau de Fruta populations exhibit weak SGS with a close-to-random spatial distribution of genotypes $\left(b_{\log }=-0.007, P=0.171 ; b_{\log }=-0.004, P=0.772\right.$, respectively). On the other hand, the genotypes of the Campina Verde population exhibit positive spatial autocorrelation indicating that groups of individuals are highly related $\left(F_{(300, \mathrm{~m})}=0.05, P<0.001\right)$. The SGS was confirmed by $S p$ statistics $(S p=0.047, P<0.001)$. This could be due to restricted pollen and seed dispersal and the anthropic pertubation history of this area; these factors are likely to contribute to the familiar structure observed among these genotypes. The genetic structure in these populations needs to be taken into account when sampling from these sites to ensure that in situ conservation strategies will be more effective.
\end{abstract}

Key words - allozymes, Cerrado, coancestry, genetic conservation, tree species

RESUMO - (Estrutura genética espacial em populações naturais de Dimorphandra mollis (Fabaceae) na região norte de Minas Gerais, Brasil). O conhecimento e o entendimento da estruturação genética intrapopulacional são importantes para o manejo e conservação dos recursos genéticos florestais, bem como para avaliar os impactos da exploração e fragmentação e estabelecer estratégias de amostragem em populações naturais. O objetivo deste estudo foi determinar a estrutura genética espacial (EGE) dentro das populações de Dimorphandra mollis Benth., visando gerar informações para a conservação genética in situ das populações naturais da espécie. Dez locos aloenzimáticos foram utilizados para estimar as frequências de 20 alelos referentes a 180 indivíduos, distribuídos em três populações naturais (Campina Verde, Pau de Fruta e Vargem da Cruz) no norte de Minas Gerais, Brasil. A diversidade genética média $\left(\hat{H}_{e}\right)$ para a espécie foi de 0,463 , considerada alta e todos os locos foram polimórficos, com média de 2,0 alelos por loco. Os genótipos de D. mollis nas populações Vargem da Cruz e Pau de Fruta encontram-se distribuídos espacialmente de maneira aleatória $\left(b_{\log }=-0,007, P=0,171 ; b_{\log }=-0,004, P=0,772\right.$, respectivamente). Por outro lado, os indivíduos da população Campina Verde apresentaram EGE positiva na menor classe de distância $\left(F_{(300, \mathrm{~m})}=0,05, P<0,001\right)$, indicando agrupamentos de indivíduos aparentados. A EGE significativa nessa população foi confirmada pela estatística $S p$, com valor de 0,047 $(P<0,001)$. Fatores como a dispersão de pólen e sementes restritas e o histórico de perturbação antrópica dessa população podem ter contribuído para esse padrão de estrutura familiar dos genótipos. Os níveis de estruturação genética detectados nas populações estudadas devem ser considerados para estratégias mais eficientes de amostragem visando à conservação genética in situ.

Palavras-chave - aloenzimas, Cerrado, coancestria, conservação genética, espécie arbórea

1. Parte da dissertação de mestrado da primeira autora no Programa de Pós-Graduação em Engenharia Florestal da Universidade Federal de Lavras, Lavras, MG, Brasil.

2. Universidade Federal de Lavras, Departamento de Biologia, Caixa Postal 3037, 37200-000 Lavras, MG, Brasil.

3. Universidade Federal do Rio Grande do Norte, Departamento de Agropecuária, 59072-970 Natal, RN, Brasil.

4. Universidade Federal de Lavras, Departamento de Ciências Florestais, Caixa Postal 3037, 37200-000 Lavras, MG, Brasil.

5. Autor para correspondência: dulce@dcf.ufla.br

\section{Introdução}

Diversos processos evolutivos e ecológicos, como a dispersão de sementes, a competição inter e intraespecífica e a heterogeneidade do ambiente podem afetar os padrões de distribuição espacial dos indivíduos dentro de uma população (Loveless \& Hamrick 1984). Além desses processos, a estrutura genética espacial (EGE) 
dentro das populações pode ser o resultado da dispersão limitada de pólen e sementes, da deriva genética local, da endogamia e da seleção, que podem favorecer um mesmo ou diferentes genótipos (Epperson 1992). Informações sobre a EGE auxiliam o entendimento dessas forças que direcionam a dinâmica genética local. Portanto, o conhecimento e o entendimento da estruturação genética intrapopulacional são importantes para 0 manejo e conservação dos recursos genéticos florestais (Epperson 1992), bem como para avaliar os impactos da exploração e fragmentação (Young \& Merriam 1994) e estabelecer estratégias de amostragem em populações naturais (Chung et al. 1998).

Vários estudos têm revelado EGE dentro de populações de espécies arbóreas (Ueno et al. 2000, Dutech et al. 2002, Martins et al. 2006). Alguns têm mostrado fraca estrutura à curta distância (Loiselle et al. 1995, Jones \& Hubbell 2006), enquanto outros não têm detectado EGE (Doligez \& Joly 1997, Epperson \& Alvarez-Buylla 1997). Essa variação pode ser devida a diferentes fatores ecológicos e genéticos operando nas populações naturais, como também a diferentes métodos de amostragem espaço-temporal e procedimentos de análises estatísticas (Smouse \& Peakall 1999, Vekemans \& Hardy 2004). Por exemplo, espécies com dispersão zoocórica podem ter diferentes estruturas espaciais entre populações (Aldrich et al. 1998, Schnabel et al. 1998). A escala e a magnitude da estrutura genética podem também diferir significativamente entre estágios de vida, com plântulas exibindo frequentemente maior estruturação dentro das populações que os adultos (Hamrick \& Nason 1996, Ng et al. 2004). Por último, novos critérios têm sido utilizados na inferência da magnitude da EGE, como a estatística $S p$, que permite a comparação entre diferentes esquemas de amostragem, populações e espécies (Vekemans \& Hardy 2004). Sendo assim, informações sobre a história de vida e mecanismos de dispersão das espécies são pré-requisitos importantes para definir a escala espacial de estudo e permitir fazer inferências sobre a distribuição espacial dos genótipos.

No Cerrado e também em outros biomas brasileiros, existem vários trabalhos relacionados à diversidade genética entre populações de espécies arbóreas. Entretanto, os estudos referentes à EGE dentro das populações naturais das espécies arbóreas são raros (Martins et al. 2006). A ausência dessas informações, aliada à falta de políticas públicas eficientes e operacionais para a conservação da natureza, aumentam o risco de extinção de populações naturais de espécies vegetais e animais. Em geral, a exploração dos recursos naturais, nesse bioma, é caracterizada pelo uso inadequado e insustentável da flora nativa. Consequentemente, a savana mais rica do mundo em biodiversidade (Myers et al. 2000, Mittermeier et al. 2004), apresenta hoje mais de $70 \%$ das áreas "altamente modificadas", devido aos desmatamentos, queimadas, uso de fertilizantes químicos e agrotóxicos; restando menos de $20 \%$ de áreas em estado conservado, consideradas críticas para a conservação (Mittermeier et al. 2004).

A espécie Dimorphandra mollis Benth. (Fabaceae), conhecida vulgarmente como favela, faveira ou fava d'anta, está entre as espécies ameaçadas pelo extrativismo no Cerrado. A espécie sintetiza em seus frutos (favas) a rutina, que é amplamente utilizada na indústria farmacêutica e de cosméticos, tanto no mercado nacional como internacional. No entanto, a espécie não é cultivada e toda a matéria prima é coletada em seu ambiente natural sem nenhum programa de manejo, caracterizando-se como extrativismo predatório. Como não há o cuidado com a reprodução da espécie, as populações naturais de Dimorphandra mollis podem ser extintas, pois a falta de manejo pode levar à redução no número de indivíduos em função da ausência de critérios para a coleta das favas (Gomes 1998). Deve-se considerar, ainda, que a coleta dos frutos sem um plano de conservação genética pode resultar na erosão da variabilidade genética, devido às restrições na disseminação de sementes e, consequentemente, com reflexos no sucesso reprodutivo e na regeneração natural da espécie.

Assim, o conhecimento da EGE das populações naturais de $D$. mollis e a compreensão de outros fatores relacionados podem ser importantes para a adoção de estratégias de manejo adequadas para a conservação genética da espécie. Para essa abordagem, marcadores aloenzimáticos têm sido comumente utilizados nas estimativas de parentesco ou coeficientes de coancestralidade (Loiselle et al. 1995, Vekemans \& Hardy 2004). Especificamente, este trabalho teve como objetivos estudar a variabilidade genética dentro e entre as populações, conhecer a distribuição espacial dos genótipos dentro das populações e gerar informações para a conservação genética in situ das populações naturais da espécie.

\section{Material e métodos}

Dimorphandra mollis Benth. (Fabaceae) - A espécie possui ampla distribuição no Cerrado, principalmente nos Estados de Goiás, Mato Grosso, Minas Gerais e São Paulo. Entre os principais usos da espécie destaca-se o medicinal, por 
meio da bioprospecção da rutina, que possui poderosa ação antioxidante, além de ser utilizada em cosméticos. A espécie é hermafrodita, sendo as flores polinizadas por pequenos insetos, entretanto, seu sistema de cruzamento ainda não está claramente definido (P.E. Oliveira, dados não publicados). A dispersão dos propágulos é feita por mamíferos não voadores (mastocoria), sendo a anta (Tapirus terrestris) considerada um importante consumidora de frutos e potencial dispersora das sementes de D. mollis (Bizerril et al. 2005). No entanto, o consumo dos frutos pode ser reduzido em função da baixa densidade desses frugívoros. Além disso, existe a possibilidade dos dispersores originais pertencerem à megafauna extinta de mamíferos da América do Sul (Bizerril et al. 2005).

Locais de estudo e amostragem - Foram estudadas três populações naturais de $D$. mollis, localizadas no Município de Jequitaí, norte do Estado de Minas Gerais. As populações estão localizadas em propriedades particulares com diferentes estádios de conservação e histórico de exploração da espécie. A população Vargem da Cruz (VC) encontra-se às margens do Rio Jequitaí, afluente do Rio São Francisco, nas coordenadas $17^{\circ} 10^{\prime} 30^{\prime \prime} \mathrm{S}$ e $44^{\circ} 29^{\prime} 55^{\prime}$ 'W e altitude de $541 \mathrm{~m}$; o tamanho da área de acordo com Scolforo et al. (2008) está estimado em torno de 9,5 ha. Segundo os relatos de moradores antigos e catadores de fava, a área era coberta por vegetação de Cerrado sensu stricto (IBGE 1993) e atualmente corresponde a uma área de extrativismo da espécie, altamente antropizada, na qual as árvores de $D$. mollis ocorrem em área de pastagens. O extrativismo dos frutos nesse local é realizado pelo proprietário, moradores meeiros e catadores da região. A densidade de indivíduos nesta área é de 10,7 ha-1 (Scolforo et al. 2008). A população Campina Verde (CV), localizada nas coordenadas $17^{\circ} 12^{\prime} 34^{\prime \prime} \mathrm{S}$ e $44^{\circ} 24^{\prime} 20^{\prime \prime} \mathrm{W}$ e altitude de $530 \mathrm{~m}$, está inserida em uma área de 18,5 ha (Scolforo et al. 2008), em que a vegetação é explorada principalmente para a produção do carvão vegetal. Nem todas as árvores de $D$. mollis são deixadas para a coleta das favas, pois observouse o corte de vários indivíduos dessa espécie. Nesta área, a densidade de indivíduos, segundo Scolforo et al. (2008), é de 13,1 ha $^{-1}$. A população Pau de Fruta (PF), nas coordenadas $17^{\circ} 13^{\prime} 20^{\prime}$ 'S e $44^{\circ} 21^{\prime} 46^{\prime \prime} \mathrm{W}$, localiza-se em uma chapada com difícil acesso e no local de maior altitude amostrado (760 m). Possui uma área com cerca de 49,5 ha (Scolforo et al. 2008). É uma região divisora de águas, que permanece com vegetação remanescente de Cerrado sensu stricto, ainda pouco explorada. Isso permite que os indivíduos da espécie se mantenham mais protegidos do extrativismo local e, com uma densidade de 24,8 indivíduos por ha (Scolforo et al. 2008). A distância entre as populações CV e VC é de $11,33 \mathrm{~km}$, entre CV e PF 5,42 km e entre VC e PF de $16,20 \mathrm{~km}$.

Em cada uma das populações foram amostrados, ao acaso, 60 indivíduos adultos com uma distância mínima de $50 \mathrm{~m}$ entre cada um, sendo que as árvores foram consideradas adultas quando se apresentavam em estágio reprodutivo. Todas as árvores amostradas foram plaqueteadas no caule com número correspondente e georreferenciadas com aparelho GPS. As amostras foliares foram colocadas em sacos plásticos identificados, acondicionadas em caixa térmica com gelo e transportadas até o laboratório, onde foram armazenadas a $75^{\circ} \mathrm{C}$. A extração das enzimas foi realizada no Laboratório de Conservação Genética de Espécies Arbóreas do Departamento de Ciências Florestais/UFLA.

Obtenção dos marcadores aloenzimáticos - As amostras foliares de cada indivíduo foram maceradas manualmente com uso de almofariz e pistilos de porcelana, mantidos resfriados durante o processo. Foram utilizados $350 \mathrm{mg}$ de tecido foliar e $1,75 \mathrm{~mL}$ da solução tampão $\mathrm{n}^{\mathrm{0}} 1$ descrita em Alfenas et al. (1998), com adição de polivinilpirrolidona-PVP e $\beta$-mercaptoetanol. Após a maceração, o produto foi centrifugado a $12.000 \mathrm{rpm}$, a $4{ }^{\circ} \mathrm{C}$, por 15 minutos. A eletroforese foi conduzida em cuba vertical, utilizando gel de poliacrilamida como suporte, sendo $4,5 \%$ o gel de concentração e $12,5 \%$ o gel de separação. Foi utilizada amperagem de $10 \mathrm{~mA}$ por gel e 300 volts, tendo a corrida eletroforética duração de aproximadamente três horas. Foram testados 15 sistemas enzimáticos de revelação, sendo selecionados os que apresentaram locos e alelos passíveis de interpretação: Álcool desidrogenase (ADH, E.C.1.1.1.1), $\beta$-Galactose desidrogenase (GLDH, E.C.1.1.1.48), Fosfatase ácida (ACP-1 eACP-2, EC 3.1.3.2), Glucose desidrogenase (GDH, E.C.1.1.1.47), Glutamato desidrogenase (GTDH, E.C.1.4.1.3), Malato desidrogenase (MDH, E.C.1.1.1.37), Peroxidase (PO, E.C.1.11.1.7), Sorbitol desidrogenase (SDH, E.C.1.1.1.14) e Xiquimato desidrogenase (SKDH, E.C.1.1.1.25). A identificação dos locos e dos alelos foi feita a partir da região mais catódica para a mais anódica. Assim, em um sistema enzimático em que dois alelomorfos são identificados, aquele com maior migração foi denominado de alelo-1 e o outro de alelo-2. Da mesma forma, foram identificados os locos presentes em cada sistema enzimático. A interpretação de cada sistema enzimático foi realizada seguindo os padrões descritos em trabalhos do gênero (Soltis \& Soltis 1989, Alfenas et al. 1998).

Diversidade e estrutura espacial dos genótipos-Avariabilidade genética de cada população foi caracterizada pela estimativa das frequências alélicas e dos índices de diversidade: número médio de alelos por loco $(\hat{A})$; porcentagem de locos polimórficos ( $P L$; critério 0,95$)$; heterozigosidades médias esperada $\left(\hat{H}_{e}\right)$ e observada $\left(\hat{H}_{o}\right)$ e índice de fixação $(\hat{f})$. Para obtenção dessas estimativas foi utilizado o software BIOSYS 2 (Swofford \& Selander 1997).

Para análise da EGE de D. mollis, os intervalos de classe de distância entre os indivíduos foram determinados testando as regras para cada intervalo conforme sugerem Hardy \& Vekemans (2002). O coeficiente de coancestria multiloco $\left(F_{i j}\right)$, com base em Loiselle et al. (1995), foi estimado entre plantas para cada uma das classes de distância usando o programa SPAGeDi versão $1.2 \mathrm{~g}$ (Hardy \& Vekemans 2002). Este coeficiente estima a probabilidade de identidade 
dos alelos em dois genes homólogos amostrados da mesma maneira. A partir de dados de marcadores genéticos estimase a coancestria relativa, que é a razão de diferenças das probabilidades de identidade por estado (Rousset 2002). A extensão da EGE foi estimada usando a estatística $S p$ (Vekemans \& Hardy 2004), que permite a comparação da magnitude da EGE entre diferentes populações. A intensidade da EGE foi calculada por $S p=-b_{l o g} /\left(1-F_{(300, \mathrm{~m})}\right)$, onde $b_{l o g}$ é a inclinação da curva de regressão do coeficiente $F_{i j}$ contra o logaritmo da distância e $F_{(300, \mathrm{~m})}$ é a média do coeficiente de coancestralidade entre os indivíduos na primeira classe de distância (0-300 m). Quando $b_{\log }=0$, aceita-se a hipótese nula de EGE aleatória. $\mathrm{O}$ erro padrão da média das estimativas foi obtido por reamostragem jackknife entre locos e, a partir dele, foram construídos intervalos de confiança a 95\% de probabilidade do coeficiente de coancestria médio estimado para cada classe de distância. A ausência de EGE foi testada dentro de cada classe de distância utilizando-se 1.000 permutações.

\section{Resultados}

Foram obtidas as frequências de 20 alelos distribuídos em dez locos aloenzimáticos para as três populações naturais de $D$. mollis (tabela 1 ). A porcentagem de locos polimórficos e o número médio de alelos por locos foram similares para todas as populações (tabela 2). A relação entre as heterozigosidades médias observadas e as esperadas forneceu índice de fixação $(\hat{f})$ não significativo para as populações.

As populações Vargem da Cruz e Pau de Fruta apresentaram distribuição aleatória dos genótipos em todas as classes de distâncias (figura 1). Os valores de coancestria para essas populações foram próximos de zero ou, negativos e não significativos (figura 1). Entretanto, a estimativa do coeficiente de coancestria na população Campina Verde na classe de distância de $0-300 \mathrm{~m}$ de $\left(F_{i j}=0,05, P<0,001\right)$ aporta EGE significativa, mostrando que existe aproximadamente $5 \%$ de probabilidade de identidade entre os alelos de pares de indivíduos nesta classe de distância. Isso indica que as árvores de $D$. mollis espacialmente próximas, nessa população, são mais aparentadas entre si do que árvores localizadas espacialmente mais distantes. Observa-se ainda que, a partir da classe de distância de 0-300 $\mathrm{m}$, houve uma acentuada queda na curva do correlograma, culminando na maior autocorrelação negativa e significativa $\left(F_{i j}=-0,03, P=0,017\right)$ nas classes de distância a partir de $1.026 \mathrm{~m}$, indicando que árvores de $D$. mollis localizadas a partir dessa classe passam a apresentar um padrão aleatório e divergente em termos de frequências alélicas. Da mesma maneira,
Tabela 1. Frequências alélicas e tamanho da amostra (n), em 10 locos aloenzimáticos, em populações naturais de Dimorphandra mollis.

Table 1. Allele frequencies and sample size $(n)$ of 10 enzymatic loci in the natural populations of Dimorphandra mollis.

\begin{tabular}{|c|c|c|c|c|}
\hline \multirow[b]{2}{*}{ Loco } & \multirow[b]{2}{*}{ Alelo } & \multicolumn{3}{|c|}{ População } \\
\hline & & $\begin{array}{c}\text { Campina } \\
\text { Verde }\end{array}$ & $\begin{array}{l}\text { Vargem } \\
\text { da Cruz }\end{array}$ & $\begin{array}{l}\text { Pau } \\
\text { de Fruta }\end{array}$ \\
\hline \multirow{3}{*}{ ACP-1 } & 1 & 0,322 & 0,750 & 0,385 \\
\hline & 2 & 0,678 & 0,250 & 0,615 \\
\hline & $n$ & 45 & 42 & 48 \\
\hline \multirow{3}{*}{ ACP-2 } & 1 & 0,744 & 0,774 & 0,781 \\
\hline & 2 & 0,256 & 0,226 & 0,219 \\
\hline & $n$ & 45 & 42 & 48 \\
\hline \multirow{3}{*}{$\mathrm{ADH}$} & 1 & 0,359 & 0,294 & 0,354 \\
\hline & 2 & 0,641 & 0,706 & 0,646 \\
\hline & $n$ & 46 & 51 & 48 \\
\hline \multirow{3}{*}{ GDH } & 1 & 0,412 & 0,458 & 0,400 \\
\hline & 2 & 0,588 & 0,542 & 0,600 \\
\hline & $n$ & 51 & 59 & 50 \\
\hline \multirow{3}{*}{ GLDH } & 1 & 0,255 & 0,387 & 0,408 \\
\hline & 2 & 0,745 & 0,613 & 0,592 \\
\hline & $n$ & 47 & 53 & 49 \\
\hline \multirow{3}{*}{ GTDH } & 1 & 0,419 & 0,417 & 0,448 \\
\hline & 2 & 0,581 & 0,583 & 0,552 \\
\hline & $n$ & 43 & 60 & 48 \\
\hline \multirow{3}{*}{$\mathrm{MDH}$} & 1 & 0,340 & 0,474 & 0,451 \\
\hline & 2 & 0,660 & 0,526 & 0,549 \\
\hline & $n$ & 50 & 57 & 51 \\
\hline \multirow{3}{*}{ PO } & 1 & 0,472 & 0,602 & 0,420 \\
\hline & 2 & 0,528 & 0,398 & 0,580 \\
\hline & $n$ & 53 & 59 & 56 \\
\hline \multirow{3}{*}{ SDH } & 1 & 0,394 & 0,407 & 0,372 \\
\hline & 2 & 0,606 & 0,593 & 0,628 \\
\hline & $n$ & 47 & 59 & 43 \\
\hline \multirow{3}{*}{ SKDH } & 1 & 0,561 & 0,500 & 0,373 \\
\hline & 2 & 0,439 & 0,500 & 0,627 \\
\hline & $n$ & 49 & 58 & 51 \\
\hline
\end{tabular}

a hipótese nula de distribuição aleatória da estrutura genética $\left(b_{\log }=0\right)$ foi rejeitada na população Campina Verde, com valor da inclinação da curva de regressão de aproximadamente $-0,045(P<0,001)$. Nas demais populações, os valores $S p$ revelaram fraca EGE e a inclinação da curva de regressão não foi significativa (tabela 2). 
Tabela 2. Índices de diversidade e estrutura genética em populações de Dimorphandra mollis. ( $n=$ tamanho amostral; $\hat{A}=$ número médio de alelos por loco; $P_{0,95}=$ porcentagem dos locos polimórficos; $\hat{H}_{o}=$ heterozigosidade observada \pm erro padrão; $\hat{H}_{e}=$ heterozigosidade esperada \pm erro padrão; $\hat{f}=$ índice de fixação de Wright; [ ] = intervalo de confiança; $b_{\log }=$ inclinação da curva de regressão do coeficiente $F_{i j} ; S p=$ intensidade da estrutura genética espacial; ns = não significativo; ${ }^{*}=P<0,05$ ).

Table 2. Genetic diversity and structure parameters in populations of Dimorphandra mollis. $(n=$ sample size; $\hat{A}=$ mean number of alleles per locus; $P_{0.95}=$ percentage of polymorphic loci; $\hat{H}_{o}=$ observed heterozygosity \pm standard error; $\hat{H}_{e}=$ expected heterozygosity \pm standard error; $\hat{f}=$ Wright's fixation index; [ ] = confidence intervals; $b_{l o g}=$ regression slope of kinship coefficients $F_{i j} ; S p$, intensity of spatial genetic structure; ns = not significantly; $*=P<0.05$ ).

\begin{tabular}{lccc}
\hline & \multicolumn{3}{c}{ População } \\
\cline { 2 - 4 }$n$ & Vargem da Cruz & Campina Verde & Pau de Fruta \\
\cline { 2 - 4 }$\hat{A}$ & 60 & 60 & 60 \\
$P_{0,95}$ & 2,0 & 2,0 & 2,0 \\
$\hat{H}_{o}$ & 100,0 & 100,0 & 100,0 \\
$\hat{H}_{e}$ & $0,494 \pm 0,058$ & $0,449 \pm 0,071$ & $0,462 \pm 0,018$ \\
$\hat{f}$ & $0,460 \pm 0,017$ & $0,459 \pm 0,014$ & $0,470 \pm 0,014$ \\
$b_{\log }$ & $-0,075^{\text {ns }}$ & $0,023^{\mathrm{ns}}$ & 0,017 ns \\
$S p$ & {$[-0,262$ a 0,134$]$} & {$[-0,253$ a 0,267$]$} & {$[-0,074$ a 0,109$]$} \\
\hline
\end{tabular}
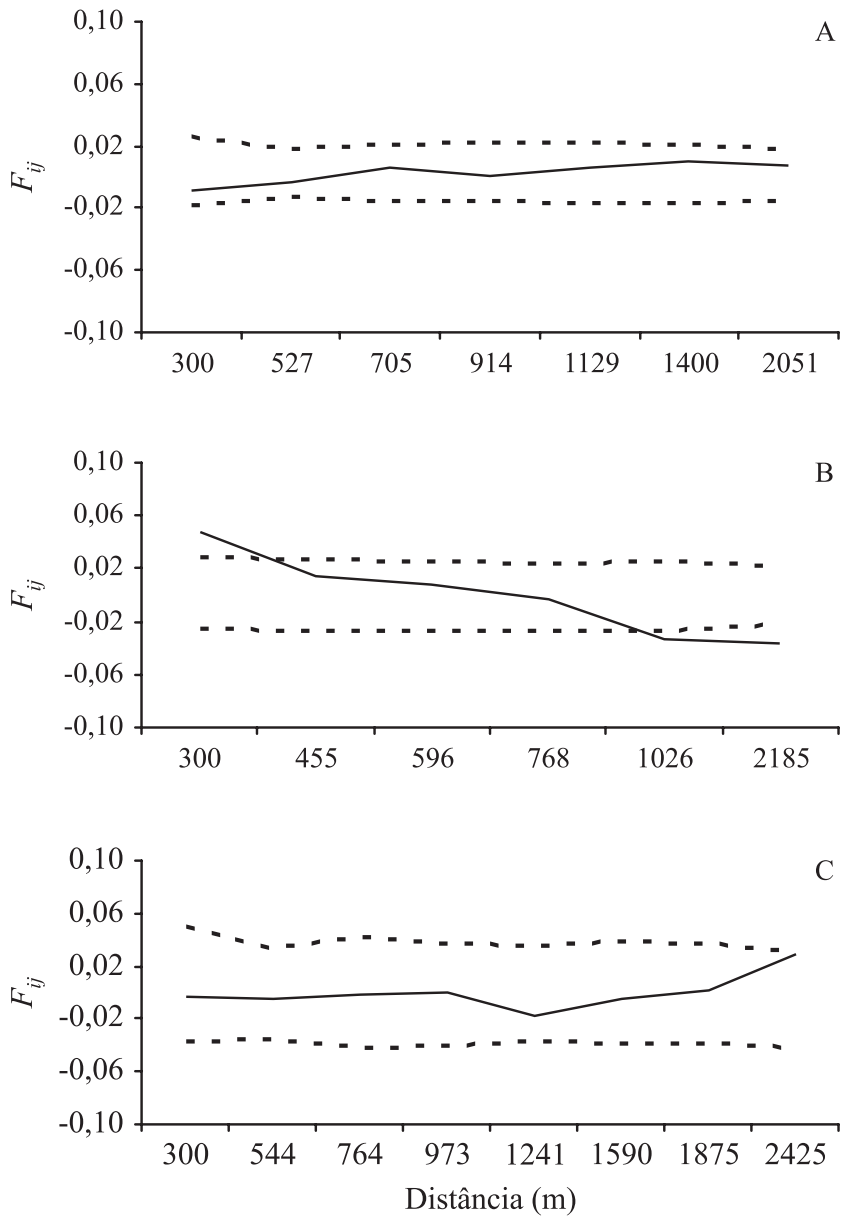

\section{Discussão}

Variabilidade genética - O percentual de locos polimórficos e o número de alelos por loco nas populações de $D$. mollis são próximos aos encontrados para várias espécies arbóreas em estudos utilizando marcadores aloenzimáticos (Botrel \& Carvalho 2004, Moraes et al. 2005, Gusson et al. 2006, Vieira \& Carvalho 2008). A ausência de alelos raros e as frequências alélicas em equidade provavelmente contribuíram para a alta diversidade genética, com heterozigosidade esperada total média $\left(\hat{H}_{e}\right)$ de 0,463 . Este valor é próximo ao relatado em populações de outras espécies arbóreas de Cerrado, mesmo exploradas pelo extrativismo tradicional

Figura 1. Correlogramas do coeficiente de coancestria $\left(F_{i j}\right)$ por classes de distâncias em três populações naturais de Dimorphandra mollis, Vargem Grande (A), Campina Verde (B) e Pau de Fruta (C). Intervalos de confiança (---) para cada valor de $\mathrm{F}_{i j}$ foram obtidos por meio de reamostragem jackknife.

Figure 1. Correlograms of kinship coefficients $\left(F_{i j}\right)$ per distance classes in three natural populations of Dimorphandra mollis, Vargem Grande (A), Campina Verde (B) e Pau de Fruta (C). Confidence intervals (---) for each $F_{i j}$-value were obtained by jackknife procedure over loci. 
dos frutos, como para Annona crassiflora $\left(\hat{H}_{e}=0,357\right.$, Telles et al. 2003) e Caryocar brasiliense $\left(\hat{H}_{e}=0,499\right.$, Melo Júnior et al. 2004).

Em condições naturais de reprodução sexual, a alta diversidade genética encontrada para $D$. mollis indicaria a possibilidade da ocorrência de numerosas novas combinações genotípicas, mantendo o potencial evolutivo e a capacidade de adaptação da espécie às possíveis mudanças ambientais. Devido aos elevados níveis de diversidade genética detectados nas populações, estratégias de manejo deveriam considerar a manutenção e a proteção dessa diversidade ao longo da paisagem estudada, visando à conservação in situ. Isso porque a exploração predatória dos frutos, impedindo a sua dispersão e consequentemente o estabelecimento natural das plântulas, poderá ocasionar a perda da diversidade genética nas próximas gerações.

As populações, aqui estudadas, apresentaram poucas diferenças entre os valores esperados e observados de heterozigosidade e, consequentemente, não apresentam desvios de panmixia $(\hat{f})$. Entretanto, as análises não permitiriam verificar a ocorrência de endogamia nas populações estudadas e seria importante efetuar estudos da estrutura genética nas próximas gerações para monitorar a manutenção ou não da diversidade genética nessas populações.

Estrutura genética espacial - A ausência de estrutura genética espacial de D. mollis nas populações Vargem da Cruz e Pau de Fruta se deve, possivelmente, a amostragem dos indivíduos a $50 \mathrm{~m}$ de distância entre eles. Serão necessárias novas amostragens a menores distâncias para verificar se a ausência de estruturação se deve realmente à distância entre os indivíduos ou ao alcance da dispersão de pólen e ou sementes. Além disso, o poder de detectar a estruturação genética é limitado ao número de genótipos utilizados, sendo essa questão relevante em futuros estudos (Cavers et al. 2005). Entretanto, vários estudos também não detectaram estruturação genética espacial em populações de algumas espécies arbóreas tropicais, associando isso à densidade de árvores adultas, competição intraespecífica, heterogeneidade ambiental e mortalidade aleatória entre plantas (Epperson \& Alvarez-Buylla 1997). A estatística $S p$ permite a comparação da magnitude da EGE entre diferentes populações e espécies (Vekemans \& Hardy 2004). Sendo assim, a EGE de $D$. mollis (média $S p=0,0194$ ) foi próxima à observada para espécies arbóreas $(S p=0,0102 \pm 0,0096)$ e polinizadas por animais $(S p=0,0171 \pm 0,0142)$ (Vekemans \& Hardy 2004).
Por outro lado, a EGE significativa dos indivíduos na população Campina Verde, na primeira classe de distância, indica agrupamentos de indivíduos provavelmente aparentados e pode ter sido consequência do extrativismo intensivo e o histórico de perturbação antrópica neste local. Essa área apresenta vegetação explorada para a produção do carvão vegetal, incluindo também indivíduos de D. mollis. Especialmente em populações de tamanho reduzido, a taxa de homozigotos tenderá a crescer com a autofecundação, assim como os cruzamentos de indivíduos aparentados que estão próximos em decorrência de dispersão de pólen e sementes à curta distância (Loveless \& Hamrick 1984). Com isso, para a população em questão, o entendimento da estruturação genética pode ser de fundamental importância para o estabelecimento de estratégias de amostragem, visando à conservação in situ. Assim, a amostragem de indivíduos distanciados a partir de $300 \mathrm{~m}$ entre si promoveria, por exemplo, uma maior probabilidade de aquisição de sementes de diferentes árvores matrizes que não são parentes, o que por sua vez, aumenta o tamanho efetivo da amostra retida. Por outro lado, apesar da população Vargem da Cruz apresentar histórico de antropização e extrativismo da espécie, para esta geração não foi observada estruturação familiar genética espacial intrapopulacional. Entretanto, como mencionado acima, a distância de $50 \mathrm{~m}$ entre os indivíduos não é adequada para estudos visando à mensuração da EGE. Estudos futuros devem investigar as consequências da depressão endogâmica nas populações naturais, assim como a capacidade das pequenas populações adaptarem às condições ambientais locais. Investigações sobre os padrões contemporâneos da estrutura genética dentro das populações e informações demográficas também são necessárias para o entendimento da estrutura genética em diferentes estágios demográficos.

Estudos de fenologia reprodutiva associados à informações demográficas, de estabelecimento das plântulas e da estrutura genética destes regenerantes da espécie no local são abordagens importantes para o futuro. Portanto, somente o acúmulo de informações pode apontar direções mais sustentáveis para o manejo, para a compreensão da dinâmica da espécie e para a efetivação de práticas conservacionistas.

Agradecimentos - Os autores agradecem ao CNPq pela concessão das bolsas de Mestrado e Produtividade em Pesquisa concedida ao primeiro e último autor; à Capes, pela concessão de bolsa de Doutorado ao terceiro autor. Agradecem à Fapemig pelo apoio financeiro concedido. Finalmente, 
agradecem a todos que, de alguma forma, participaram na realização deste trabalho.

\section{Referências bibliográficas}

ALDRICH, P.R., HAMRICK, J.L., CHAVARRIAGE, P. \& KOCHERT, G. 1998. Microsatellite analysis of demographic genetic structure in fragmented populations of the tropical tree Symphonia globulifera. Molecular Ecology 7:933-944.

ALFENAS, A.C., BRUNE, W., OLIVEIRA, J.R., ALONSO, S.K. \& SCORTICHINI, M. 1998. Extração de proteínas para eletroforese. In Eletroforese de isoenzimas e proteínas afins: fundamentos e aplicações em plantas e microrganismos. (A.C. Alfenas, ed.). UFV, Viçosa, p.85-114.

BIZERRIL, M.X.A., RODRIGUES, F.H.G. \& HASS, A. 2005. Fruit consumption and seed dispersal of Dimorphandra mollis Benth. (Leguminosae) by the lowland tapir in the Cerrado of Central Brazil. Brazilian Journal of Biology 65:407-413.

BOTREL, M.C.G. \& CARVALHO, D. 2004. Variabilidade isoenzimática em populações naturais de jacarandá paulista (Machaerium villosum Vog.). Revista Brasileira de Botânica 4:621-627.

CAVERS, S., DEGEN, B., CARON, H., LEMES, M.R., MARGIS, R., SALGUEIRO, F., \& LOWE, A.J. 2005. Optimal sampling strategy for estimation of spatial genetic structure in tree populations. Heredity 95:281289.

CHUNG, M.Y., CHUNG, G.M. \& CHUNG, M.G. 1998. Spatial genetic structure in populations of Cymbidium goeringii (Orchidaceae). Genes \& Genetic Systems 73:281-285.

DOLIGEZ, A. \& JOLY, H.I. 1997. Genetic diversity and spatial structure within a natural stand of a tropical tree species, Carapa procera (Meliaceae), in French Guiana. Heredity 79:72-82.

DUTECH, C., SEITER, J., PETRONELli, P., JOLY, H.I. \& JARNE, P. 2002. Evidence of low gene flow in a neotropical clustered tree species in two rain forest stands of French Guiana. Molecular Ecology 11:725738.

EPPERSON, B.K. 1992. Spatial structure of genetic variation within populations of forest trees. New Forests 6:257278.

EPPERSON, B.K. \& ALVAREZ-BUYLLA, E.R. 1997. Limited seed dispersal and genetic structure in life stages of Cecropia obtusifolia. Evolution 51: 275-282.

GOMES, L.J. 1998. Extrativismo e comercialização da fava d'anta (Dimorphandra sp.) estudo de caso na região de cerrado de Minas Gerais. Dissertação de mestrado, Universidade Federal de Lavras, Lavras, MG.
GUSSON, E., SEBBENN, A.M. \& KAGEYAMA, P.Y. 2006. Sistema de reprodução em populações de Eschweilera ovata (Cambess.) Miers. Revista Árvore 30:491-502.

HAMRICK, J.L. \& NASON, J.D. 1996. Consequences of dispersal in plants. In Population dynamics in ecological space and time. (O.E. Rhodes Jr., R.K. Chesser \& M.H. Smith, eds.). University of Chicago Press, Chicago.

HARDY, O. \& VEKEMANS, X. 2002. SPAGeDi 1. 2: a versatile computer program to analyze spatial genetic structure at the individual or population levels. Molecular Ecology Notes 2:618-620. Disponível em: http://www.ulb.ac.be/sciences/lagev/software.html. (acessed 13/07/2009)

IBGE - Instituto Brasileiro de Geografia e Estatística. 1993. Classificação da vegetação brasileira. IBGE, São Paulo.

JONES, F.A. \& HUBBELL, S.P. 2006. Demographic spatial genetic structure of the Neotropical tree, Jacaranda copaia. Molecular Ecology 15:3205-3217.

LOISELLE, B.A., SORK, V.L., NASON, J.D. \& GRAHAM, C. 1995. Spatial genetic structure of a tropical understory shrub, Psychotria officinalis (Rubiaceae). American Journal of Botany 11:1420-1425.

LOVELESS, M.D. \& HAMRICK, J.L. 1984. Ecological determinants of genetic structure in plant populations. Annual Review of Ecology and Systematics 15:65-95.

MARTINS, K., CHAVES, L.J., BUSO, G.S.C. \& KAGEYAMA, P.Y. 2006. Mating system and fine-scale spatial genetic structure of Solanum lycocarpum St. Hill. (Solanaceae) in the Brazilian Cerrado. Conservation Genetics 7:957-969.

MELO JUNIOR, A.F., CARVALHO, D., PÓVOA, J.S.R. \& BEAZORTI, E. 2004. Estrutura genética de populações naturais de pequizeiro (Caryocar brasiliense Camb.). Scientia Forestalis 66:56-65.

MITTERMEIER, R.A., GIL,P.R., HOFFMANN, M., PILGRIM, J.D., BROOKS, T., MITTERMEIER, C.G. \& FONSECA, G.A.B. 2004. Hotspots revisited: earth's biologically richest and most endangered terrestrial ecoregions. CEMEX/Conservation International, México.

MORAES, M.L.T., KAGEYAMA, P.Y. \& SEBBENN A.M. 2005. Diversidade e estrutura genética espacial em duas populações de Myracrodruon urundeuva Fr. All. sob diferentes condições antrópicas. Revista Árvore 29:281-289.

MYERS, N., MITTERMEIER, R.A., MITTERMEIER, C.G., FONSECA, G.A.B. \& KENT, J. 2000. Biodiversity hotspots for conservation priorities. Nature 403:853-858.

NG, K.K.S., LEE, S.L. \& KOH, C.L. 2004. Spatial structure and genetic diversity of two tropical tree species with contrasting breeding systems and different ploidy levels. Molecular Ecology 13:657-669.

ROUSSET, F. 2002. Inbreeding and relatedness coefficients: what do they measures? Heredity 88:371-380. 
SCHNABEL, A., NASON, J.D. \& HAMRICK, J.L. 1998. Understanding the population genetic structure of Gleditsia triacanthos L.: seed dispersal and variation in female reproductive success. Molecular Ecology 7: 819-832.

SCOLFORO, J.R, MELLO, J.M., OLIVEIRA, A.D. (EDS.). 2008. Inventário florestal de Minas Gerais: cerrado-florística, estrutura, diversidade, similariadade, distribuição diamétrica e de altura, volumetria, tendências de crescimento e áreas aptas para manejo florestal. Universidade Federal de Lavras, Lavras.

SMOUSE, P.E. \& PEAKALL, R. 1999. Spatial autocorrelation analysis of individual multiallele and multilocus genetic structure. Heredity 82:561-573.

SOLTIS, D.E. \& SOLTIS, P.S. 1989. Isoenzymes in plant biology. Dioscorides Press, Portland.

SWOFFORD, D.L. \& SELANDER, R.B. 1997. Biosys-2. a computer program for the analysis of allelic variation in population genetics and biochemical systematics. University of Illinois, Urbana.
TELLES, M.P.C., VALVA, F.D., BANDEIRA, L.F. \& COELHO, A.S.G. 2003. Caracterização genética de populações naturais de araticunzeiro (Annona crassiflora Mart. - Annonaceae) no Estado de Goiás. Revista Brasileira de Botânica 26:123-129.

UENO, S., TOMURA, N., YOSHIMARU, H., MANABE, T. \& YAMAMOTO, S. 2000. Genetic structure of Camellia japonica L. in an old-growth evergreen forest, Tsushima, Japan. Molecular Ecology 9:647-656.

VEKEMANS, X. \& HARDY, O.J. 2004. New insights from fine-scale spatial genetic structure analyses in plant populations. Molecular Ecology 13:921-935.

VIEIRA, F.A. \& CARVALHO, D. 2008. Genetic structure of an insect-pollinated and bird-dispersed tropical tree in vegetation fragments and corridors: implications for conservation. Biodiversity and Conservation 17: 2305-2321.

YOUNG, A.G. \& MERRIAM, H.G. 1994. Effects of forest fragmentation on the spatial genetic structure of Acer saccharum Marsh. (sugar maple) populations. Heredity 72:201-208. 